\title{
Does the core collapse in globular clusters onset before than commonly believed?
}

\author{
Marco Merafina* \\ Department of Physics, University of Rome "La Sapienza", p.le A. Moro 5, I-00185 Rome (Italy) \\ E-mail: marce.merafinadromal.infn.it
}

\begin{abstract}
An analysis of the structural parameters of globular clusters (GCs) carried out by using data contained in the Harris Catalogue indicates a clear missing coincidence between the maximum of the $W_{0}$-distribution and the onset of gravothermal instability. This question, firstly outlined by Katz, is a still open problem because the coincidence is necessary if considering the dynamical evolution of the GCs population. In order to tackle the problem, a selfconsistent thermodynamical model of GCs is developed by considering a different approach with respect to the King one in calculating the distribution function which must be solution of the Fokker-Planck equation. Requiring a Boltzmann form of the distribution function expressed by an Hamiltonian function leads to a solution containing additional term in the total energy. This new term, called effective potential, simulates the effects of the tidal forces induced by the hosting galaxy on the phase space limitation in the velocities of stars. The influence of the effective potential on the virial theorem will be described, together with the related consequences on the insurgence of thermodynamical instability. Interesting results implying a different critical point for the onset of gravothermal catastrophe now in accordance with observational data are presented, as well numerical simulations confirming the presence of the effective potential with the predicted form.
\end{abstract}

XII Multifrequency Behaviour of High Energy Cosmic Sources Workshop

12-17 June, 2017

Palermo, Italy

${ }^{*}$ Speaker. 


\section{Introduction}

The development of theory in stellar dynamics, especially in the part devoted to globular clusters, was delayed, not to say stopped, by the increased power of calculators since at least 25 years.

Nowadays, the analysis is mainly performed by N-body numerical simulations only focused to reproduce observational data. This approach in general misses some theoretical aspects necessary in understanding specific phenomena in the evolution of GCs, like the energy equipartition among stars, the mass segregation problem and the gravothermal instability.

It is well known that dynamical evolution of GCs is strongly affected by collisions among the stars, making favourable a statistical mechanics approach in order to study this important phenomenon. GCs are stellar systems with masses within the interval $10^{4}-10^{6} M_{\odot}$, containing a number of stars of the order of $10^{5}$. For their spherical symmetry, there is the possibility to test the evolution of a GC by studying a classical single mass King model [山] in relation to thermodynamical instability phenomena.

In the evolution of GCs, stellar encounters strongly contribute in phase space mixing of stellar orbits and thermodynamics plays a centrale role in the gravitational equilibrium and stability of these clusters, due to relatively small relaxation time with respect to their old absolute age which ranges between 10 to 13 Gyr. Therefore, GCs are the best possible laboratory to test the effects of thermodynamics in a selfgravitating system where the collisions among stars are important in the dynamical evolution.

On the other hand, the observations of the luminosity profiles of different GCs [వ] show similar curves depending only on different values of the star concentration, giving the possibility to fit them by an empirical law and suggesting a unique distribution function for the whole sample of clusters [四].

Thus, the evolution of GCs can be descripted by a sequence of thermodynamic equilibrium configurations with different equilibrium parameters [B]]. This approach is equivalent to consider small thermodynamic transformations which keep constant the functional form of the velocity distribution of stars like in the framework of Boltzmann statistical mechanics, with the relevant difference that the nature of collisions is described in the Fokker-Planck approximation. The gravitational equilibrium models are exactly the same obtained by King [四, but in the analysis of the thermodynamical instabilities important differences are found, relevant in the evolution of GCs.

GCs are probably the only "point of contact" between gravity and thermodynamics and a serious analysis of this aspect leads to consequences in the calculation of the onset of gravothermal instability inducing the core collapse of the cluster, making able to conclude that this collapse occurs earlier than commonly believed.

\section{The observed GC population in the Milky Way}

An important question, not yet solved in the last forty years, is connected with the maximum of the $W_{0}$-distribution of Milky Way GCs with respect to the critical point related to the onset of gravothermal catastrophe. This problem was firstly outlined by Katz [ [⿴囗十] with the comparison of the rms value of the central gravitational potential of the clusters distribution $\left(W_{0}=6.9\right)$, well approximating the value corresponding to the peak of the distribution, with the value $\left(W_{0}=7.4\right)$ 
corresponding to the theoretical onset of gravothermal catastrophe. Katz obtained these values for 51 GCs by using the central escape velocity $v_{e s c}$ and the central one-dimensional velocity dispersion $v_{\text {disp }}$ tabulated by Peterson \& King [ []] and Peterson [ [ $]$ ]. The values of $W_{0}^{\text {rms }}$ and $W_{0 C}$ were expected to be coinciding because GCs population had enough time to undergo the gravothermal catastrophe and, therefore, the $W_{0}$-distribution of GCs should peak exactly in correspondence to the critical value. In fact, the primeval Gaussian distribution, approaching the critical value during the evolution, deforms in a non-symmetric Gaussian curve due to the effect of gravothermal catastrophe which progressively subtracts the collapsed GCs with values of $W_{0}$ larger than the critical value (See Fig.W). For these reasons, the resulting distribution must be asymmetric and must present a maximum in correspondence of the onset of gravothermal instability. Nevertheless the

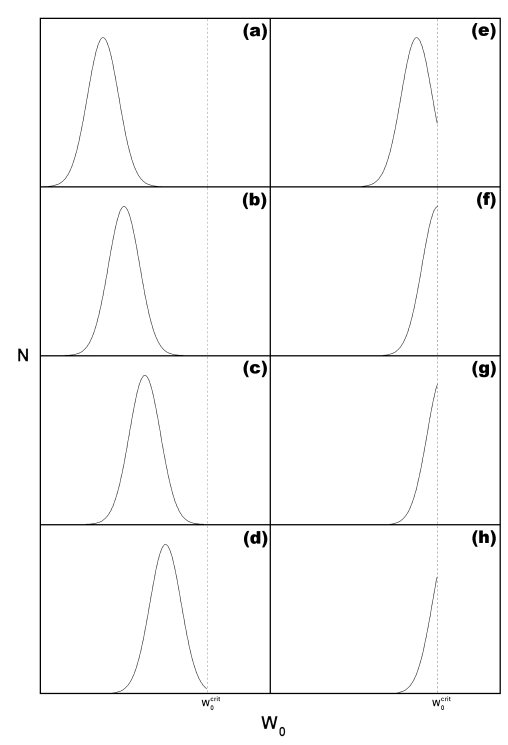

Figure 1: Evolution of GCs population with respect to $W_{0}$ from a) to h). After $f$ ) the maximum of the population always coincides with the critical point characterizing the onset of gravothermal catastrophe.

values are significantly different and also the analysis of new data from Harris Catalogue on Milky Way GCs confirm this discrepancy. In fact, according with a statistical study of a group of 124 not post-core-collapsed globular clusters, selected from a sample of 157 objects included in the latest edition of Harris Catalogue [ $[\square]$, the critical value $W_{0 C}=7.4$ results not compatible with the actual calculated value corresponding to the maximum of the $W_{0}$-distribution $W_{0}^{\max }=6.87$. This value is deducible by considering the skewness of the $W_{0}$-distribution obtained from the observational data which is related to theoretical parameters of the asymmetric Gauss distribution, in particular the ratio $B$ between the left and right standard deviation, useful to determinate the value of $W_{0}^{\max }$ corresponding to the peak of the distribution. The gap between the current stability limit $W_{0 C}=7.4$ and the peak value of the distribution at $W_{0}^{\max }=6.87$ is significantly large, and only a $W_{0}$-distribution with a skewness completely different $(B \ll 1)$ from one given by observational data $(B=0.54675)$ could justify such a stability limit. (see Fig.(D).

Several models can be considered in the equilibrium as well in thermodynamical analysis of GCs, involving different distribution functions. Among these, in the framework of the Boltzmann distribution function, we can mention the Lynden-Bell \& Wood model [[] describing an isother- 


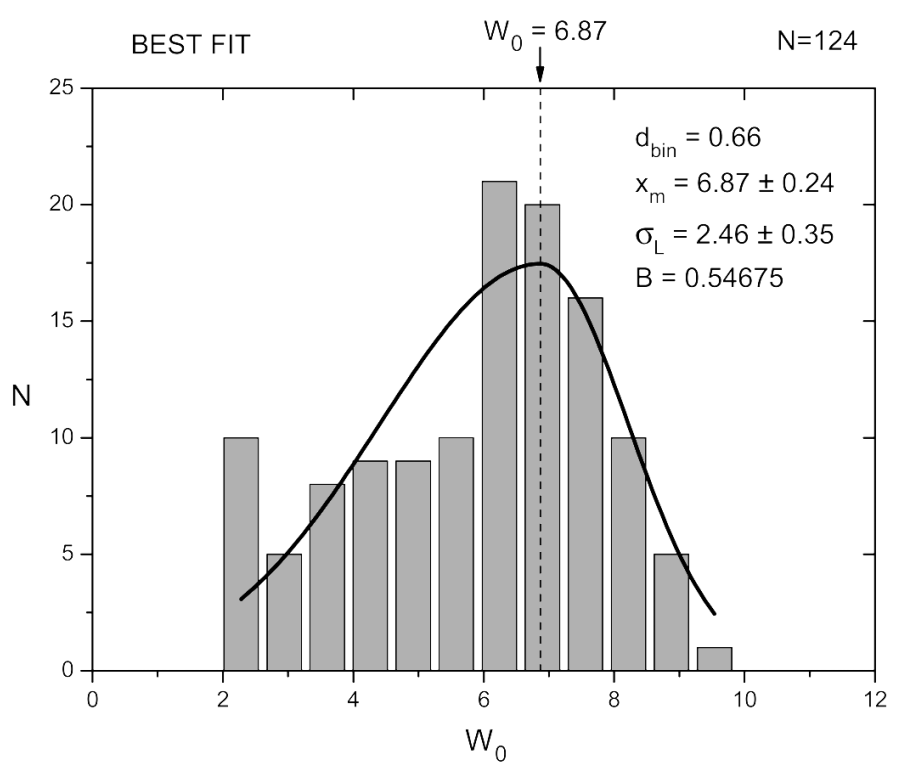

Figure 2: MW GCs distribution at different values of $W_{0}$ based on the latest edition (2010) of the Harris Catalogue by considering the concentration $c$ of each GC [D].

mal sphere confined in a box and also equilibrium models with truncated Maxwellian distribution function considered in [Q]. In these two cases, the equilibrium configurations well describe density profiles of the inner regions in GCs. Differently, in the identification of the onset of gravothermal instability, they give results which are not in accordance with the observational data, even if with values which are smaller than one obtained by Katz. In the Lynden-Bell \& Wood case we have $W_{0 C}=6.55$, while in the case of models with truncated Maxwellian distribution function we obtain $W_{0 C}=6.42[$ [Q] .

Then, it is necessary to reconsider the calculation of the Fokker-Planck solution focusing the attention on the definition of the distribution function and taking into account the request of a thermodynamic equilibrium distribution which maximizes the entropy. The mandatory choice is considering a Boltzmann form of the distribution and solving with respect to the Hamiltonian.

\section{Reconsidering the calculation of the Fokker-Planck equation}

In order to understand if the onset of gravothermal catastrophe is correctly performed, the calculation of the solution of the Fokker-Planck equation has been reconsidered, starting from the assumption that the distribution in energy must have a Boltzmann form to correctly define the entropy as the logarithm of the statistical weight of macroscopic states. For solving the equation in terms of Hamiltonian of the single star, the part not depending on time of the Fokker-Planck equation, in the form introduced by Spitzer \& Harm [ए]], must be considered

$$
\frac{d}{d x}\left[G(x)\left(\frac{d g(x)}{d x}+2 x g(x)\right)\right]+\lambda x^{2} g(x)=0
$$


where

$$
G(x)=\frac{4}{\sqrt{\pi} x} \int_{0}^{x} e^{-y^{2}} y^{2} d y,
$$

while $x=v / \sqrt{2} \sigma$ and $\sigma=\sqrt{k \theta / m}$ are the dimensionless velocity and the Boltzmann velocity dispersion, respectively. The parameter $\lambda$ is the fractional loss rate of the stellar evaporation suffered by the cluster [W].

We are looking for a solution of the form $g(x)=A e^{-H(x) / k \theta}$, where $H(x)=H_{0}(x)+H_{1}(x)$ is the Hamiltonian of the single star and $H_{1}(x)=0$ when $\lambda=0$. So that, being $\lambda \ll 1$, we can write at first order

$$
\exp \left[-H_{1}(\lambda, x) / k \theta\right] \simeq 1-\left.\frac{\lambda}{k \theta} \frac{\partial H_{1}}{\partial \lambda}\right|_{\lambda=0} .
$$

Inserting $g(x)$ in Eq.(B.Cl), equating the terms with the same power of $\lambda$ and neglecting the higher order terms to $\lambda$ we obtain

$$
\left.\frac{\partial H_{1}}{\partial \lambda}\right|_{\lambda=0}=\frac{\sqrt{\pi}}{8} k \theta\left(e^{x^{2}}-1\right),
$$

and, finally, we get the Hamiltonian form as

$$
H(x)=k \theta\left\{x^{2}-\ln \left[1-\frac{\sqrt{\pi}}{8} \lambda\left(e^{x^{2}}-1\right)\right]\right\} .
$$

The additional term to kinetic energy in the obtained Hamiltonian function is named "effective potential". This term vanishes when the parameter $\lambda$, connected to star evaporation, becomes equal to zero and the distribution tends to Boltzmann one.

\section{Discussion}

With the resolution of the Fokker-Planck equation we obtained in the Hamiltonian of the distribution an additional term, named "effective potential", which is the effect of the equilibrium established by two competitive processes: one due to collisions among stars with an exchanging of energy which tends to modify the distribution by driving it to the Boltzmann one with the formation of a tail at large energy; one, in opposition, due to evaporation of stars as result of the presence of tidal forces induced by the hosting galaxy that removes continuously the stars and prevents the formation of the tail, maintaining a cutoff energy and a limited phase space. These two competitive effects keep unchanged the form of the energy distribution function during the dynamical evolution of the GCs, namely that we can consider as a "thermodynamical equilibrium" from the astrophysical point of view, with the collisions ruled by the Fokker-Planck approximation.

The presence of the effective potential does not affect the internal dynamics of stars and sum of kinetic and gravitational energy remains constant in the motion between two consecutive collisions, as well the effective potential. The result is that the Hamiltonian (total energy) remains a constant of motion and the dynamical problem of the single mass orbit keep unchanged. Nevertheless, the presence of the effective potential has important consequences from the thermodynamical point of view resulting in the formation of regions with negative and positive specific heat which guarantee the evolution of the system towards the gravothermal instability [ए2].

Moreover, the additional (positive) contribution of the effective potential on the total energy $E_{t o t}=E_{k i n}+E_{g r}+E_{e f f}$, considering also the virial condition $2 E_{k i n}+E_{g r}=0$, implies that $E_{t o t}=$ 
$-E_{k i n}+E_{\text {eff }}$. The term $E_{\text {eff }}$ is the counterpart of the term $3 P_{b} V$ obtained by Lynden-Bell \& Wood [8] for the isothermal sphere, characterized by infinite radius and, therefore, confined by a spherical wall surrounded by a termal bath. Differently, $E_{\text {eff }}$ results when the phase space limitation is correctly performed on the momentum space.

Adopting the new expression of the virial theorem, the behavior of the total energy $E_{t o t}$ (in units of $M v_{0}^{2}$ ) is investigated as a function of the quantity $\sigma^{2}=k \theta / m$ (in units of $v_{0}^{2}$ ). The quantity $v_{0}$ is a normalization constant with the dimensions of a velocity. The obtained curve is shown in Fig. [3, where the most important results are also summarized. It is parameterized in terms of increasing values of $W_{0}$ as it spirals inwards.

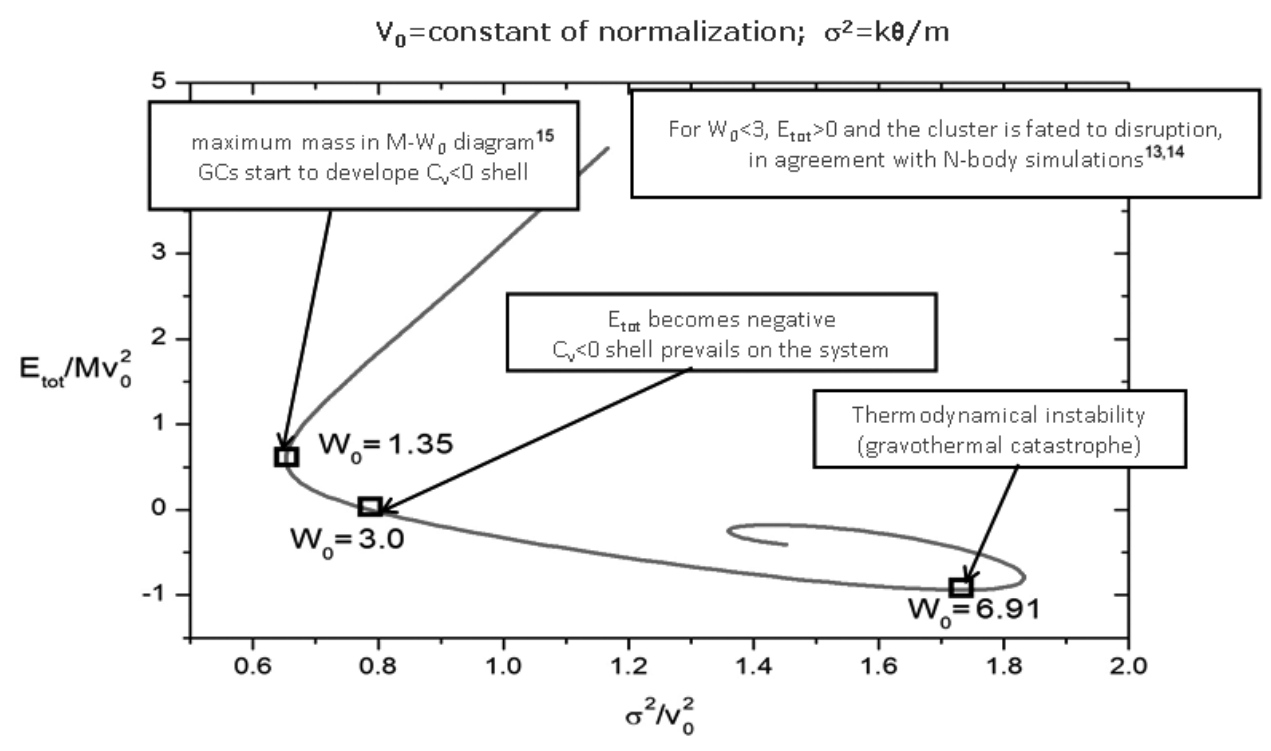

Figure 3: Caloric curve taking into account the contribution of the effective potential.

The critical point which sets the beginning of the gravothermal instability is located where the curve has the horizontal tangent point. The presence of $E_{\text {eff }}$ affects the caloric curve and change the critical point to a value which corresponds to the maximum of the GC distribution (from $W_{O C}=7.4$ to $W_{0 C}=6.91$ ) [[3], in complete agreement with observational data.

The forty-year open question appears to be definitely solved taking into account the effects due to the presence of the effective potential. This implies that the core collapse in GCs onsets before than commonly believed.

Another important result derived by this treatment is the threshold at $W_{0}=3$ corresponding to the zero total energy. Clusters with lesser values of $W_{0}$ are fated to disruption, because the tidal forces are prevalent to the gravitational ones. This result can explain observative data and N-body simulations going to the same conclusions [[4, [5]].

Finally, it is worth to stress the importance of the value $W_{0}=1.35$ corresponding to the appearance of regions with negative specific heat in the cluster. This value is reached in correspondence to the maximum mass in the $M-W_{0}$ diagram of the equilibrium configurations [ए6]. Systems with $W_{0}<1.35$ cannot evolve, being unable to exchange heat between negative and positive specific 
heat regions, since they have only a unique positive specific heat region all over the equilibrium configuration.

\section{Effective potential and N-body simulations}

Waiting for observative data on transverse velocities of stars in GCs, enabling to test the model and the presence of effective potential in real systems, a N-body simulation for a model with $N=262144$ stars was performed. In order to extract informations valid for the entire cluster we considered the total energy of each star and calculated the total number of stars of equal sum $E$ of gravitational and kinetic energy, comparing the obtained distribution with the Boltzmann form with the Hamiltonian $H$ above carried out. The effective potential $\psi$ of a single star can be expressed in dimensionless form as

$$
\Delta=-\ln \left(1-e^{\Sigma-W_{\infty}}\right),
$$

where $\Delta=\psi / k \theta, W_{\infty}=\varphi_{R} / \sigma^{2}$ is the dimensionless form of the gravitational potential at the surface of the cluster, while $\Sigma=E / k \theta$ is the dimensionless sum of the gravitational $m \varphi$ and kinetic energy $\varepsilon$ of a single star. Using the number of stars at fixed $\Sigma$ allows to obtain the value of $H$ and consequently the effective potential by subtracting kinetic and gravitational energy to the total energy calculated. The results are summarized in Fig.t.

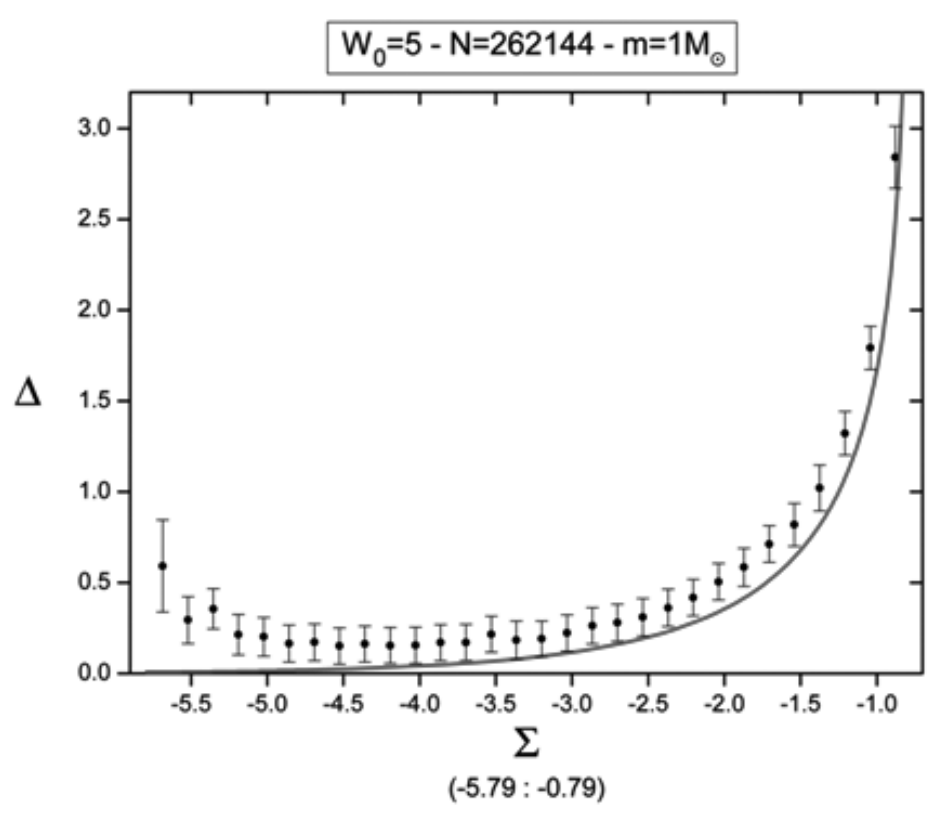

Figure 4: Effective potential by the N-body simulation. The continuous curve represents the theoretical prediction.

A very good accordance with the expected curve has been obtained. The small disagreement is due to the transition from continuous (theory) to discrete ( $\mathrm{N}$-body simulation) system, depending on the choice of the radial-energy mesh for individuating the sample of stars with same energy. This disagreement becomes more relevant at low energies, especially at the center of the cluster, for lack of stars at zero kinetic energy. 


\section{Conclusions}

The additional contribution of the effective potential allows to construct selfconsistent models admitting regions with positive and negative heat capacity which can exchange energy and produce gravothermal instability, without the necessity to assume an external bath as in the Lynden-Bell \& Wood model.

A new critical value for the onset of gravothermal instability has been obtained. This value coincides with one related to the peak of the GCs distribution, removing the unexpected difference outlined by Katz. This is an observational evidence of the presence of the effective potential, confirmed by the analysis of data of more than 150 GCs of the Harris Catalogue.

The possibility to obtain also positive values of the total energy of the clusters, due to presence of the effective potential, gives the opportunity to explain the conditions of disruption of the clusters at very small values of $W_{0}$ where the tidal forces prevail on the gravitational ones. These conditions are in accordance with the observations and several N-body simulations.

$\mathrm{N}$-body simulations are developed in order to test the presence of the effective potential. The presence is confirmed and also the behaviour is in very good accordance with the predicted one. The recent possibility of measuring transverse velocities of the stars in GCs opens important perspectives in order to test the presence of the effective potential also in real systems for supporting the validity of the model.

\section{References}

[1] King, I.R. 1966, AJ, 71, 64

[2] King, I.R. 1962, AJ, 67, 471

[3] Horwitz, G., Katz, J. 1977, ApJ, 211, 226

[4] Katz, J. 1980, MNRAS, 190, 497

[5] Peterson, C.J., King, I.R. 1975, AJ, 80, 427

[6] Peterson, C.J. 1976, AJ, 81, 617

[7] Harris, W.E. 1996, AJ, 112, 1487

[8] Lynden-Bell, D., Wood, R. 1968, MNRAS, 138, 495

[9] Bisnovatyi-Kogan, G.S., Merafina, M. 2006, ApJ, 653, 1445

[10] Spitzer, L., Harm, R. 1958, ApJ, 127, 544

[11] King, I.R. 1965, AJ, 70, 376

[12] Merafina, M., Vitantoni, D. 2014, Acta Polytechnica CTU Proc., 1, 231

[13] Piscicchia, K., Merafina, M., Proceedings of XIV Marcel Grossmann Meeting on General Relativity, in press

[14] Chernoff, D.F., Weinberg, M.D. 1990, ApJ, 351, 121

[15] Fukushige, T., Heggie, D.C. 1995, MNRAS, 276, 206

[16] Merafina, M., Ruffini, R. 1989 A\&A, 221, 4 